\title{
Análisis de la punción de prótesis intravasculares recubiertas tras el rescate de la fístula arteriovenosa después de $\mathbf{1 2}$ meses de experiencia
}

\author{
Victoria Eugenia Gómez López, Carolina Muñoz Macías, Rafael Casas Cuesta, Óscar Calle Mafla, Rodolfo \\ Crespo Montero
}

UGC Nefrología. Unidad de hemodiálisis. Hospital U. Reina Sofía. Córdoba. España

\section{Resumen \\ Objetivos: Evaluar la eficacia a medio plazo de una nueva prótesis intravascular (VIABAHN ${ }^{\circledR}$ ), y su seguri- dad, analizando la incidencia de eventos adversos aso- ciados a su punción.}

Pacientes y Método: Se estudiaron 20 pacientes, a los que se les implantó VIABAHN ${ }^{\circledR}$, por pseudoaneurismas con trombosis, úlceras en las zonas de punción, fracaso de angioplastia o reestenosis en menos de 6 meses.

Se estudió: Parámetros de la FAV, permeabilidad primaria y secundaria (6 y 12 meses), presencia de complicaciones y control del deterioro de la prótesis mediante flebografía a los 3 y 6 meses.

Para la punción se esperó un periodo de 4 semanas.

Resultados: Se analizó un periodo de $15 \pm 9.4$ meses. La media de los parámetros funcionales de la FAV durante la hemodiálisis fue: Fs: $362.5 \pm 43.3 \mathrm{ml} / \mathrm{min}$, PA: $-209.5 \pm 35.6 \mathrm{mmHg}$, PV: $215.8 \pm 34.5 \mathrm{mmHg}, \mathrm{Kt}$ $55 \pm 5.4$ I y Recirculación $12.7 \pm 3.7 \%$.

A los 6 meses el 66\% (IC 95\% 54-77) de los pacientes tenía permeabilidad primaria y el $77 \%$ (IC $95 \%$ 67-87) tenía permeabilidad secundaria. A los 12 meses el 38\% (IC 95\% 24-52) permeabilidad primaria y el $76 \%$ (IC 95\% 66-87) permeabilidad secundaria.

Correspondencia:

Rafael Casas Cuesta

Servicio de Hemodiálisis

Hospital Universitario Reina Sofía

Avda. Menéndez Pidal s/n. 14004. Córdoba

E-mail: rafcasas@ono.com
No se observaron eventos adversos relacionados con la punción, ni se objetivó radiológicamente deterioro en las prótesis. Hubo un episodio de infección que requirió la retirada de la prótesis.

Conclusiones: La prótesis vascular recubierta VIABAHN $^{\circledR}$ es eficaz y segura en el rescate de las FAVs nativas estenosadas y/o trombosadas, ya que proporciona excelentes parámetros de diálisis y alta permeabilidad primaria y secundaria, sin que su punción repetida se asocie a complicaciones.

PALABRAS CLAVE: fístula arteriovenosa autóloga; prótesis intravascular; stent; punción fístula arteriovenosa.

Analysis of puncture of coated intravascular prostheses after salvage of arteriovenous fistula, after 12 months of experience

Aim: To evaluate the medium-term efficacy of a new intravascular prosthesis (VIABAHN ${ }^{\circledR}$ ), and its safety, by analyzing the incidence of adverse events associated with its puncture.

Patients and method: Twenty patients, with VIABA$\mathrm{HN}^{\circledR}$ implanted, were studied for pseudoaneurysms with thrombosis, ulcers in the puncture sites, failure of angioplasty or restenosis in less than 6 months. Parameters of AVF, primary and secondary patency ( 6 and 12 months), presence of complications and control of prosthesis deterioration by phlebography at 3 and 6 months were studied. For the puncture, a period of 4 weeks was expected. 
Results: A period of $15 \pm 9.4$ months was analyzed. The mean of the functional parameters of the AVF during hemodialysis was: blood flow rate: $362.5 \pm 43.3 \mathrm{ml}$ / min, Arterial Pressure: $-209.5 \pm 35.6 \mathrm{mmHg}$, Venous Pressure: $215.8 \pm 34.5 \mathrm{mmHg}$, Kt $55 \pm 5.4 \mathrm{I}$ and recirculation $12.7 \pm 3.7 \%$. At 6 months, $66 \%$ (95\% CI: 54-77) of the patients had primary permeability and $77 \%$ (95\% CI 67-87) had secondary permeability. At 12 months 38\% (IC 95\% 24-52) primary permeability and $76 \%$ (IC 95\% 66-87) secondary permeability. No adverse events related to the puncture, nor radiologically objectified deterioration in prostheses were observed. There was an episode of infection requiring removal of the prosthesis.

Conclusión: The VIABAHN ${ }^{\circledR}$ coated vascular prosthesis is effective and safe in the rescue of stented and / or thrombosed native AVFs, as it provides excellent dialysis parameters and high primary and secondary permeability, without repeated puncture associated with complications.

KEYWORDS: autologous arteriovenous fistula; intravascular prosthesis; stent; arteriovenous fistula puncture.

\section{Introducción}

En la actualidad, la fístula arteriovenosa nativa (FAVn) es el acceso vascular de elección para hemodiálisis (HD), seguido del injerto protésico o fístula arteriovenosa protésica (FAVp) y como último recurso el catéter venoso central, ya que se ha demostrado que es el acceso que menos complicaciones presenta, incidiendo esto de forma determinante en la calidad de vida del paciente y en su morbimortalidad ${ }^{1,2,3}$.

Una de las complicaciones más conocidas que aparece en la FAVn y FAVp como consecuencia de las punciones repetidas, sobre todo cuando se puncionan en las mismas zonas, es la aparición de pseudoaneurismas, y como consecuencia del flujo turbulento que se origina en su interior, la formación de estenosis tanto a nivel de la anastomosis como a la salida de las zonas dilatadas 4 .

Por tanto, la presencia de pseudoaneurismas suponen una situación de riesgo para el paciente, ya que las estenosis pueden disminuir el flujo intraacceso favoreciendo la trombosis y consecuente pérdida del acceso, o pueden favorecer el aumento de presión intraacceso favoreciendo aún más el desarrollo del pseudoaneurisma pudiendo llegar incluso a su rotura ${ }^{5}$.

El tratamiento de los pseudoaneurismas ha sido tradicionalmente el quirúrgico, resecando o ligando el tramo venoso afectado, y reemplazándolo por un segmento de prótesis vascular, habitualmente de Politetrafluoroetileno $\mathrm{PTFE}^{6}$, necesitándose un periodo post intervención de al menos 3-4 semanas de maduración, antes de su utilización. Se trata pues de una técnica invasiva, que además impide el uso del acceso vascular durante un tiempo más o menos prolongado ${ }^{7}$.

Por otro lado, el tratamiento habitual de las estenosis, la angioplastia intraluminal con balón (ATP), no siempre es eficaz y no está exenta de complicaciones agudas como roturas vasculares, presentando además una alta incidencia de reestenosis ${ }^{8-11}$.

Actualmente y gracias al desarrollo tecnológico que se ha experimentado en las técnicas endovasculares con la aparición de nuevos materiales como los stent recubiertos de politetrafluoroetileno (PTFE), se ha experimentado un avance en el tratamiento de estas complicaciones. Se trata de prótesis tubulares de estructura metálica distensible recubiertas de PTFE, y que se colocan en el interior del vaso trombosado 0 estenosado, permitiendo el flujo sanguíneo en ese tramo. Se implantan con técnicas mínimamente invasivas y son capaces de excluir el tramo venoso dilatado al mismo tiempo que se tratan las posibles estenosis existentes.

Estas prótesis, aunque están diseñadas para otros fines distintos a la reparación de FAVs y por tanto su punción no se contempla en las especificaciones de su ficha técnica, en la actualidad se están puncionando cuando no existen zonas de punción alternativas, obteniéndose diferentes resultados según las series estudiadas $5,10,12,13,14$.

Una de las más recientes es el VIABAHN ${ }^{\circledR}$ una nueva prótesis intravascular, que se ha empezado a implantar en estos casos, en los que el tratamiento habitual no consigue repermeabilizar el tramo venoso afectado, y aunque tampoco su ficha técnica contempla la punción repetida, ante la ausencia de zonas de punción alternativas, en nuestro Servicio, y de acuerdo con la Unidad de Radiología Intervencionista de nuestro Centro, hemos comenzado a puncionarlos, y estamos actualmente evaluando sus resultados. 
Por consiguiente, el objetivo del presente estudio ha sido evaluar la eficacia a medio plazo de la prótesis intravascular Viabahn ${ }^{\circledR}$ en su uso como acceso vascular para HD y su seguridad, analizando la incidencia de eventos adversos asociados a su punción.

\section{Pacientes y Método}

Se estudiaron entre enero de 2014 y octubre de 2016 a 20 pacientes en HD de la UGC Nefrología del Hospital Universitario Reina Sofía de Córdoba a los que se les había implantado prótesis recubiertas Viabahn ${ }^{\circledR}$ para la reparación/recuperación de FAVs trombosadas en presencia de pseudoaneurismas, por fracaso de las ATPs al tratar estenosis o por reestenosis tras ATPs en menos de 6 meses, o por presentar úlceras en las zonas de punción.

Las prótesis se empezaron a puncionar a partir de la $4^{a}$ semana tras su implantación con agujas $16 \mathrm{G}$ y por personal "experimentado".

Durante este periodo de "maduración" de la prótesis, 5 pacientes precisaron para la HD la implantación de un catéter venoso central como acceso vascular, al carecer de zonas de punción alternativas para la punción distintas a la prótesis, mientras otros 15 pacientes se dializaron en unipunción, puncionando la aguja en el tramo de vena nativa existente entre la anastomosis de la FAV y el inicio de la prótesis, alojando el extremo de la aguja en la luz de la endoprótesis.

Posteriormente al periodo de "maduración" de las prótesis, los pacientes se dializaron en bipunción, puncionando las prótesis con agujas 16G.

Durante el periodo de estudio se realizaron flebografías a los 3 y 6 meses (figuras 1 y 2), para controlar el posible deterioro de las prótesis derivado de las punciones repetidas y se estudiaron las siguientes variables:

- Incidencia de infecciones o eventos adversos asociados a la punción.

- Permeabilidad primaria y secundaria a los 6 y 12 meses.

- Parámetros funcionales dinámicos del circuito de HD (Caída de la presión en la línea arterial (PA), presión venosa (PV) en monitores 5008 (Fresenius ${ }^{\circledR}$ ) y Recirculación con Monitor de Temperatura Sanguínea $\left(\mathrm{BTM}^{\circledR}\right)$.

- Eficacia de la HD (Kt).

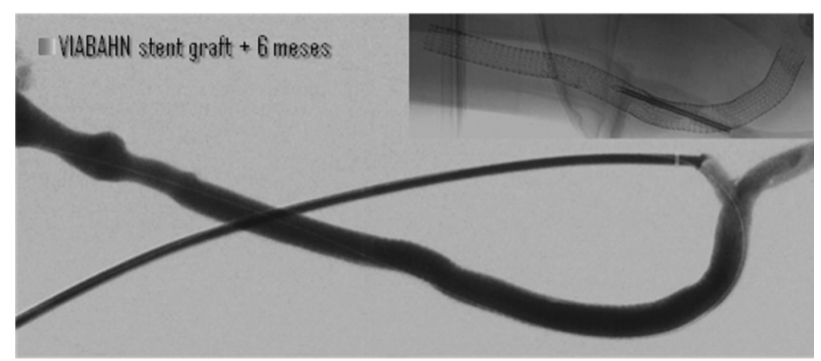

Figura 1.

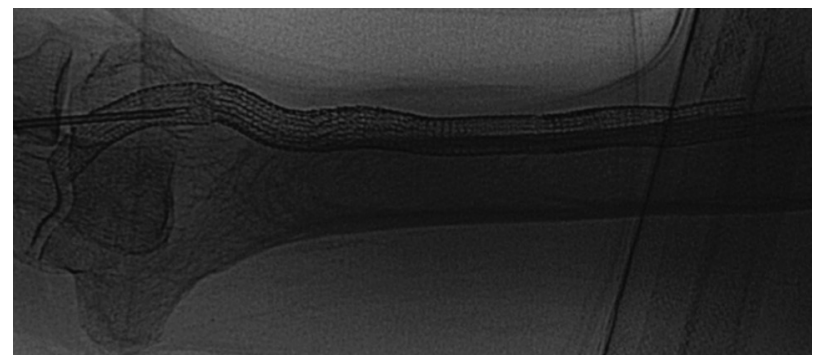

Figura 2.

\section{Análisis estadístico}

Para el análisis estadístico se realizó un análisis descriptivo, presentando las variables cualitativas como una distribución de frecuencias y las variables cuantitativas como la media+la desviación típica. Se realizó en un paquete estadístico SPSS 15.0.

\section{Resultados}

Se analizó un periodo de seguimiento de 15+9.4 meses en el que se registró 1 episodio de infección que requirió la retirada del injerto, y no se registraron eventos adversos ni se objetivaron radiológicamente deterioros derivados de las punciones.

La media de los parámetros funcionales del circuito de $\mathrm{HD}$ al final del periodo de seguimiento fueron: $\mathrm{Qb}$ : $362.5 \pm 43.3 \mathrm{ml} / \mathrm{min}, \mathrm{PA}:-209.5 \pm 35.6 \mathrm{mmHg}, \mathrm{PV}$ : $215.8 \pm 34.5 \mathrm{mmHg}, \mathrm{Kt}: 55 \pm 5.4$ I y Recirculación: $12.7 \pm 3.7 \%$.

Los datos sobre la permeabilidad, primaria y secundaria de los injertos durante el periodo de estudio, se presentan en la tabla 1 .

Tabla 1. Permeabilidad del Viabahn ${ }^{\circledR}$ a los 6 y 12 meses.

\begin{tabular}{|lcc|}
\hline & 6 meses & 12 meses \\
\hline Permeabilidad Primaria & $66 \%$ & $38 \%$ \\
\hline Permeabilidad secundaria & $77 \%$ & $76 \%$ \\
\hline
\end{tabular}




\section{Discusión}

Al comparar nuestros resultados de permeabilidad con lo descrito en las guías SEN/SEDEN sobre FAVn y con estudios previos realizados sobre FAVp de PTFE, observamos que se acercan a lo descrito en las guías para la permeabilidad de las $F A V n$, y que superan lo descrito para la permeabilidad de las FAVp de PTFE ${ }^{3,15}$.

Aunque nuestros resultados de permeabilidad a los 12 meses parecen indicar la eficacia de la implantación de estas endoprótesis, que pensamos se puede deber a que la estructura del Viabahn ${ }^{\circledR}$ puede proteger en cierta medida ante la aparición de complicaciones como los pseudoaneurismas y/o estenosis, creemos que sería necesario aumentar la muestra del estudio y el tiempo de seguimiento para confirmar estos resultados preliminares, ya que estudios recientes, con una muestra más amplia que la nuestra, han obtenido buenos resultados iniciales de permeabilidad sin objetivar un aumento significativo en la permeablilidad global; observando además, altas tasas de reestenosis y trombosis no asociadas a las prótesis, concluyendo que este tipo de endoprótesis no mejora la supervivencia media del acceso vascular ${ }^{16}$.

Otro resultado a destacar es la infección registrada durante el periodo de estudio, y que requirió la retirada de la prótesis a pesar de haber aplicado estrictas medidas de asepsia en el manejo de estos accesos, siguiendo las recomendaciones de las diferentes guías, aunque hay que reflejar que esta endoprótesis se puncionó antes de cumplir el periodo de 4 semanas de maduración.

De nuestra experiencia, destaca también la ausencia de eventos adversos derivados de las punciones, los cual puede deberse a que, debido a las dificultades de punción que presentan estas prótesis por su estructura metálica, siempre han sido puncionadas por el personal más experimentado de la unidad, en el intento de minimizar todo lo posible el riesgo de punciones fallidas que conllevaran riesgo de hematomas, desgarros de la prótesis y manipulaciones de aguja que pudieran favorecer sangrados e infecciones ${ }^{17}$.

Respecto al deterioro de las prótesis por las punciones repetidas, destacar que a diferencia de otros autores que describen deterioros importantes ${ }^{12}$, en nuestro grupo no hemos detectado radiológicamente deterioros importantes, ya que en solo 2 pacientes se detectaron microroturas del stent sin fuga de contraste. Pensamos que esto puede ser debido al calibre de agujas utilizado (16G); a la técnica de punción esca-
Ionada, y a que el material metálico con el que está fabricado el Viabahn ${ }^{\circledR}$, el Nitinol, aleación de niquel y titanio, es más elástico que otros materiales empleados en otras prótesis, y se deforma con la punción y vuelve a su forma original con mayor facilidad tras la retirada de la aguja ${ }^{5}$.

Sobre la eficacia de este tipo de acceso vascular, nuestros datos muestran que se han mantenido en niveles aceptables los parámetros dinámicos del circuito de HD y la recirculación del acceso, al mismo tiempo que han permitido dar al paciente una diálisis adecuada, teniendo siempre en cuenta, que se han puncionado con agujas 16G.

Por tanto, a la vista de nuestros resultados, y con la debida cautela, dado lo reducido de nuestra muestra y el tiempo de seguimiento, podemos concluir que la prótesis vascular recubierta Viabahn ${ }^{\circledR}$, es eficaz y segura en el rescate de $F A V n$ estenosadas y/o trombosadas, ya que proporciona excelentes parámetros de diálisis y alta permeabilidad primaria y secundaria al año, sin que su punción repetida se asocie a complicaciones.

\section{Conflicto de intereses}

Los autores declaran estar libres de cualquier asociación personal o comercial que puedan suponer un conflicto de interés relacionado con el contenido de este trabajo

\begin{tabular}{|c|}
\hline Recibido: 28 julio 2017 \\
Revisado: 16 agosto 2017 \\
Modificado: 20 agosto 2017 \\
Aceptado: 25 agosto 2017 \\
\hline
\end{tabular}




\section{Bibliografía}

1. K/D0QI Clinical Practice Guidelines and Clinical Practice Recommendations for 2006 Updates: hemodialysis adequacy, peritoneal dialysis adequacy, vascular access. Am J Kidney Dis 2006; 48: S1-S322.

2. Al-Jaishi AA, Oliver MJ, Thomas SM, Lok CE, Zhang JC, Garg AX, et al. Patency rates of the arteriovenous fistula for hemodialysis: a systematic review and meta-analysis. Am J Kidney Dis. 2014 Mar;63(3):464-78.

3. Ravani P, Palmer SC, Oliver MJ, Quin RR, Mac Rae JM, Tai DJ, et al. Association betwen hemodialysis Access type and clinical outcomes: a systematic review. J Am Soc Nefrol. 2013 febr; 24(3):465-73.

4. Rodríguez $H E$, Leon $L$, Schalch $P$, Labropoulous $N$, Borge $M$, Kalman PG. Arterivenous access: managing common problems. Perspect Vasc Surg. Endovasc Ther. 2005; 17:155-166.

5. Pandolfe LR, Malamis AP, Pierce K, Borge MA. Treatment of hemodialysis graft pseudoaneuryms with stent grafts:institutional experience and review of the literature. Seminars in interventional radiology. 2009; 26 (2): 89-95.

6. Belli S, Parlakgumus A, Colakoglu T, Ezer A, Yildirim $S$, et al. Surgical treatment modalities for complicated aneurysms and pseudoaneurysms of arteriovenous fistulas. J Vasc Access. 2012; 13(4):438-45.

7. Saran R, Dykstra DM, Pisoni RL, Akiba T, Akizawa $\mathrm{T}$, et al. Timing of first annulations and vascular failure in haemodialysis an analysis of practice patterns at dialysis facilities in the DOPPS. Nephrol Dial Transplant. 2004; 19: 2334-2340.

8. Turmel Rodrigues $L$, Pengolan J, Blanchier $D$, Abaza $M$, Birmelé $B$, et al. Insufficient dialysis shunts: improved long-term patency rates wich close hemodynamic monitoring, repeated percutaneus ballon angioplasty, and stent placement. Radiology. 1993; 187:273-278.

9. Kanterman RY, Vesely TM, Pilgram TK, Guy BW, Windus DW, Picus D. Dialysis access grafts: Anatomic location of venous stenosis and results of angioplasty. Radiology. 1995; 196:582.
10. Sapoval MR, Turmel-Rodrigues LA, Raynaud AC, et al. Cragg covered stents in hemodialysis access: initial and midterm results. J Vasc Interv Radiol 1996; 7:335-342.

11. Vogel PM, Parise C. SMART stent for salvage of hemodialysis access graft. J Vasc Interv Radiol. 2004; 15:1051-1060.

12. Hausegger KA, Tiessenhausen $K$, Klimpfinger M, Raith J, Hauser H, Tauss J. Aneurysms of hemodialysis access graft: treatment with covered stents: a report of three cases. Cardiovasc Intervent Radiol 1998; 21: 334-337.

13. Barshes NR, Annambhotla S, Kougias $P$, Bechara C, Huynh TT, et al. Endovascular repair of hemodialysis graft-related pseudoaneurysm: An alternative treatement strategy in salvaging failing dialysis access. Vascular and endovascular Surgery. 2008; 42 (3):228-234.

14. Silas AM, Bettman MA. Utility of covered stents for revision of aging failing synthetic hemodialysis grafts: a report of three cases. Cardivasc Intervent Radiol. 2003; 26: 550-553.

15. Huber TS, Carter JW, Carter RL, Seeger JM. Patency of autogenous and polytetrafluoroethylene upper extremity arteriovenous hemodialysis accesses: A systematic review. J Vasc Surg. 2003;38:1005-1011.

16. Schmelter C, Raab U, Lazarus F, Ruppert V, Vorwerk D. Outcomes ot AV fistulas and AV Grafts after interventional stent-graft deployment in haemodialysis patiens. Cardiovasc Intervent Radiol. 2015; 38(4):878-8.

17. Nassar GM, Ayus JC. Infectious complication of the hemodialysis access. Kidney Int. 2001; 60: 1-13. 